\title{
Development of a duplex reverse transcription recombinase-aided amplification assay for respiratory syncytial virus incorporating an internal control
}

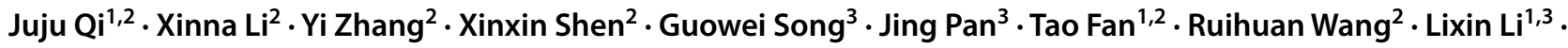 \\ Xuejun $\mathrm{Ma}^{2}$
}

Received: 26 January 2019 / Accepted: 6 March 2019 / Published online: 3 May 2019

(c) Springer-Verlag GmbH Austria, part of Springer Nature 2019

\begin{abstract}
Human respiratory syncytial virus (RSV) is a common viral pathogen that causes lower respiratory tract infections in infants and children globally. In this study, we developed a duplex reverse transcription recombinase-aided amplification (duplexrtRAA) assay containing an internal control in a single closed tube for the detection of human RSV. The internal control in the amplification effectively eliminated false-negative results and ensured the accuracy of the duplex-rtRAA system. We first developed and evaluated a universal singleplex-rtRAA assay for RSV. The sensitivity of this assay for RSV was determined as 4.4 copies per reaction, and the specificity was $100 \%$. Next, a duplex-rtRAA assay with an internal control was established. The sensitivity of the duplex-rtRAA assay approached 5.0 copies per reaction, and no cross-reaction with other common respiratory viruses was observed. The two detection methods (singleplex-rtRAA and duplex-rtRAA) developed in this study were used to test 278 clinical specimens, and the results showed absolute consistency with RSV RT-qPCR analysis, demonstrating $100 \%$ diagnostic sensitivity and specificity. These data indicate that the duplex-rtRAA has great potential for the rapid detection of RSV with a high sensitivity.
\end{abstract}

Handling Editor: William G. Dundon.

Juju Qi, Xinna Li and Yi Zhang contributed equally to the article.

Electronic supplementary material The online version of this article (https://doi.org/10.1007/s00705-019-04230-z) contains supplementary material, which is available to authorized users.

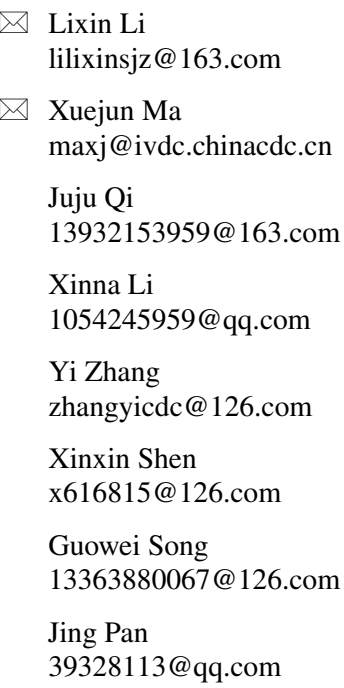

Acute respiratory tract infections (ARTIs) are one of the main causes of outpatient visits, hospitalization, and death, especially in children, the elderly and immunocompromised individuals [1-3]. The major pathogens causing ARTIs in infants and young children are viruses, the most frequently reported of which include respiratory syncytial virus (RSV),

Tao Fan

hugengkun@163.com

Ruihuan Wang

15286855786@163.com

1 Hebei Medical University, 361 East Zhongshan Road, Shijiazhuang 050031, Hebei, China

2 Key Laboratory for Medical Virology, National Health and Family Planning Commission, National Institute for Viral Disease Control and Prevention, Chinese Center for Disease Control and Prevention, No. 155 Changbai Road, Changping District, Beijing 102206, China

3 Myasthenia Gravis Research Institute, The First Hospital of Shijiazhuang, 36 Fanxi Road, Shijiazhuang 050011, Hebei, China 
adenovirus, influenza viruses $\mathrm{A}$ and $\mathrm{B}$, rhinovirus, and parainfluenza virus. Some research has indicated that pneumonia and bronchitis in infants is more frequently caused by RSV than by any other pathogenic microorganisms in most areas [4]. RSV is an enveloped virus belonging to the family Paramyxoviridae that has a single-stranded nonsegmented negative-sense RNA. There are two distinct groups (A and B) of RSV, which are distinguished by antigenic and genetic variations [5]. Respiratory infection of RSV may lead to bronchitis, bronchial pneumonia and severe pneumonia and can even be life-threatening. RSV pneumonia is difficult to distinguish from pneumonia caused by influenza virus or adenovirus based on clinical symptoms. Currently, there are still no effective vaccines and treatments [6]. Therefore, rapid and accurate detection of RSV is of great importance not only for monitoring and controlling the prevalence of RSV but also for providing reliable laboratory evidence for early clinical diagnosis and selection of the appropriate treatment $[7,8]$.

Conventional laboratory diagnosis of RSV infection involves the use of cell culture, direct immunofluorescence assays (DFA), rapid antigen detection tests (RADTs), reverse transcription polymerase chain reaction (RT-PCR), real-time RT-PCR and multiplex RT-PCR [9-13]. Although cell culture is considered the gold standard for virus detection with high specificity, its low sensitivity and time required limit its application in clinical diagnosis. Serological detection also has the disadvantage of low sensitivity. In recent years, RT-PCR, with high sensitivity and specificity, has gradually become a mainstream method of pathogen detection [14]. However, RT-PCR requires specialized technical expertise, a dedicated laboratory, and expensive thermocycling equipment, and it is also time-consuming and costly, which is not conducive to its widespread use in conventional laboratories and in low-resource areas. The isothermal amplification technique, which was developed in recent years, provides an alternative to PCR technology [15]. Examples of isothermal nucleic acid amplification techniques include nucleicacid-sequence-based amplification (NASBA) [16, 17], loop-mediated isothermal amplification (LAMP) [18, 19], helicase-dependent amplification (HDA) [20], recombinase polymerase amplification (RPA) [21], and recombinaseaided amplification (RAA) [22, 23]. These methods do not require thermocycling. Unlike other isothermal amplification techniques, RAA does not require an initial denaturation step to separate double-stranded DNA. The basic RAA assay includes two specific primers and three core proteins (recombinase UvsX from Escherichia coli, DNA polymerase, and single-strand DNA binding protein [SSB]), and the reaction is carried out at $37-42{ }^{\circ} \mathrm{C}$ within 30 minutes. The real-time RAA reaction system additionally includes a novel exo oligonucleotide probe and exonuclease III in addition to the basic system.
In this study, we developed a duplex-rtRAA assay for the detection of RSV with a competitive internal control, and the specificity, sensitivity, and clinical performance of this assay were evaluated.

A total of 278 clinical specimens of nasopharyngeal secretions were collected from hospitalized patients with acute viral infection of the lower respiratory tract at the Children's Hospital of Hebei Province (China) between January and March, 2018. Of these patients, 58 (40.75\%) were female and $121(59.25 \%)$ were male, and their ages ranged from 1 month to 10 years. In this study, 278 specimens were tested retrospectively using a GeXP-based multiplex RTPCR assay method established previously in our laboratory [24], of which 102 were positive for RSV, 77 were positive for other respiratory viruses, and 99 were negative. Viral RNA was extracted using a Viral RNA/DNA Isolation Kit (Tianlong, Suzhou, China) according to the manufacturer's instructions. The extracts were eluted in $70 \mu \mathrm{l}$ of elution buffer and stored at $-80{ }^{\circ} \mathrm{C}$ until further processing. The study was conducted with the approval of the Institutional Review Boards of the National Institute for Viral Disease Control and Prevention, Center for Disease Control and Prevention of China. All Caregivers of the children were informed and signed a written informed consent.

We download all of the available complete genome sequences of RSV from the National Center for Biotechnology Information database (NCBI) and performed a multiple sequence alignment using Vector NTI version 11.5.1 to identify genome regions that are highly conserved in all genotypes of RSV for the design of universal primers and probes. The RSV primers RSV-F and, RSV-R and the RSV probe RSVP-0, targeting the L-gene region of RSV, were used in the follow-up study. An RSV internal control (RSVIC) probe (P-IC) was also designed to target an internal control (IC) template in the RSV duplex RAA assay. All oligonucleotides used in this study are listed in Table 1 . The RSV probe and RSVIC probe were labelled with the FAM and HEX fluorophore, respectively. All primers and probes were designed with the aid of Oligo7, and no nonspecific sequences were found. All of the oligonucleotides were purified by HPLC and synthesized by Sangon Biotech (Shanghai, China).

The RT-PCR primers (Table 1) used to amplify the RSV plasmid were designed in this study and used to amplify a 1462-bp fragment of the RSV genome extending from (nt 13451 to nt 14913, GenBank accession no. KY982517), which was then verified by Sanger sequencing and cloned into the pClone007 vector by TsingKe Biotech Corp (Beijing, China). The internal control (IC) template sequence was a 373-bp recombinant sequence consisting of two fragments from rose rosette virus (nt 199-249, GenBank accession no. HQ891913.1) and RSV (nt 13948-14299, GenBank accession no. KY982517). The RSV fragment contained the common primer sequences and other sequences that served 
Table 1 The oligonucleotides used for RT-PCR, rtRAA, and RT-qPCR

\begin{tabular}{|c|c|c|c|c|c|}
\hline & Primer/probe & Sequence $\left(5^{\prime}-3^{\prime}\right)$ & $\begin{array}{l}\text { Primer } \\
\text { length (bp) }\end{array}$ & Product size (bp) & Reference \\
\hline \multirow[t]{2}{*}{ RT-PCR } & PCR-F & CCCTTGGGTTGTTAACATAGA & 21 & 1463 & This study \\
\hline & PCR-R & TCATTGGTTGTCAAGCTGTTT & 21 & & \\
\hline \multirow[t]{4}{*}{ rtRAA } & RSV-F & TCCYAATTGTATAGCATTCATAGGTGAAGGAGC & 33 & 206 & This study \\
\hline & RSV-R & TTGCATCTGTAGCAGGAATGGTYAAATTYTCAC & 33 & & \\
\hline & $\mathrm{RSV}-\mathrm{P} 0^{\mathrm{a}}$ & $\begin{array}{l}\text { CATCCTGATATAAGATATATTTACAGAAG[T(FAM- } \\
\text { dT)][Y(THF)][T(BHQ-dT)]GAAAGATTGCAA } \\
\text { TGA[C3-spacer] }\end{array}$ & 47 & & \\
\hline & $\mathrm{P}-\mathrm{IC}^{\mathrm{a}}$ & $\begin{array}{l}\text { GTAAGGTGCTAGACTAAAATTGTTGGGAC[T(HEX- } \\
\text { dT)][T(THF)][T(BHQ-dT)]GAATCTCTGAAGTAA } \\
\text { AAGG[C3-spacer] }\end{array}$ & 51 & & \\
\hline \multirow[t]{3}{*}{ RT-qPCR } & qPCR-F & CACWGAAGATGCWAATCATAAATTCA & 26 & 89 & {$[25]$} \\
\hline & qPCR-R & GTATYTTTATRGTGTCTTCYCTTCCTAACC & 30 & & \\
\hline & qPCR-P & FAM-TAATAGGTATGTTATATGCKATGTC-BHQ & 25 & & \\
\hline
\end{tabular}

Probe modifications: FAM, 6-carboxyfluorescein; HEX, 5-hexachlorofluorescein; THF, tetrahydrofuran; BHQ, Black Hole Quencher; C3-spacer, 3'phosphate blocker

as a backbone, while only the probe-binding region of RSV was replaced by a rose rosette virus fragment. Thus, the IC sequence contained the same primer and template sequences as the target (RSV plasmid), but the probe-binding region was different (Fig. S1). The recombinant plasmid containing the IC sequence (RSVIC) was constructed by TsingKe Biotech Corp (Beijing, China) by synthesizing the IC region and cloning it into the pUC57 vector. The plasmid DNA was quantified using a Qubit dsDNA BR/HR assay kit with a Qubit 2.0 fluorometer (Life Technologies, Warrington, UK) according to manufacturer's instructions. Then, the RSV recombinant plasmid standard was serially diluted from $10^{5}$ to $10^{0}$ copies $/ \mu \mathrm{L}$, and the RSVIC plasmid was serial diluted to $10,000,5000,1000,500,100$ copies $/ \mu \mathrm{L}$.

The universal singleplex-rtRAA assay was performed in a 50- $\mu \mathrm{L}$ reaction volume using an RT-RAA exo kit (Qitian, Jiangsu, China) according to the manufacturer's instructions. Briefly, $25 \mu \mathrm{L}$ of rehydration buffer, $16.7 \mu \mathrm{L}$ of DNase-free water, $2.1 \mu \mathrm{L}$ of each primer $(10 \mu \mathrm{M})$, and $0.6 \mu \mathrm{L}$ of probe $(10 \mu \mathrm{M})$ were added to RT-RAA tubes containing a dried enzyme pellet (SSB, UvsX, DNA polymerase, exonuclease III). Then, we covered the tube and mixed the contents by tapping lightly. After brief centrifugation, $2.5 \mu \mathrm{L}$ of $280 \mathrm{mM}$ magnesium acetate and $1 \mu \mathrm{L}$ of RNA template or $1 \mu \mathrm{L}$ of DNase-free water for the no-template control (NTC) were added to the tube. The reaction mix was incubated in a QT-B6100 constant-temperature oscillating mixing instrument (Qitian, Jiangsu, China) for 4 minutes and then transferred to a QT-F1620 fluorescence detection instrument with FAM and HEX dual channel detection equipment (Qitian, Jiangsu, China) at $39.0{ }^{\circ} \mathrm{C}$ for $30 \mathrm{~min}$. NTC reactions were performed in parallel.
Based on the singleplex-rtRAA assay, we tried a duplexrtRAA assay by introducing an internal control. The duplexrtRAA assay was also performed in a $50-\mu \mathrm{L}$ reaction volume using an RT exo kit (Qitian, Jiangsu, China). The reaction mixture contained a dried enzyme pellet (SSB, UvsX, DNA polymerase, exonuclease III), $25 \mu \mathrm{L}$ of rehydration buffer, $15.1 \mu \mathrm{L}$ of DNase-free water, $2.1 \mu \mathrm{L}$ of each primer $(10 \mu \mathrm{M})$, $0.6 \mu \mathrm{L}$ of probe $(10 \mu \mathrm{M}), 0.6 \mu \mathrm{L}$ of RSVIC probe $(10 \mu \mathrm{M})$, and $1 \mu \mathrm{L}$ of RSVIC plasmid (100 copies per reaction). The remaining reaction components included $1 \mu \mathrm{L}$ RNA template or $1 \mu \mathrm{l}$ of DNase-free water (NTC) and $2.5 \mu \mathrm{L}$ of $280 \mathrm{mM}$ magnesium acetate. Reaction conditions and procedures were the same as in the singleplex-rtRAA assay reactions described above. The concentration of the RSVIC plasmid was optimized to ensure that the amplification of the RSVIC would not affect the amplification of the RSV template and that the amplification signal could be detected at each dilution of the RSV plasmid. We used a series of dilutions of the RSV plasmid $\left(10^{5}-10^{0}\right.$ copies per reaction) to determine the optimal concentration of the RSVIC. Each duplex-rtRAA assay was repeated eight times, and NTC reactions were performed for each run as described previously.

The sensitivity of the singleplex-rtRAA assay and duplexrtRAA assay was evaluated using serial tenfold dilutions $\left(10^{4}, 10^{3}, 10^{2}, 10^{1}\right.$ and $10^{0}$ copies $\left./ \mu \mathrm{L}\right)$ of RSV recombinant plasmid standards and RSV-negative clinical specimens $(176 / 278)$ were used to evaluate specificity. These samples were positive for one of the following pathogens: adenovirus (ADV), influenza virus A or B (FluA or B), human rhinovirus (HRV), parainfluenza virus (PIV), coronavirus (COV), human metapneumovirus (HMPV), and human bocavirus $(\mathrm{HBoV})$. Each assay was repeated eight times. 
A total of 278 clinical specimens of nasopharyngeal secretions were tested by both the singleplex-rtRAA and duplex-rtRAA methods, and the results were compared to those obtained using a previously reported RT-qPCR method [25].

A dilution range of $1 \times 10^{4}-1 \times 10^{0}$ copies per reaction of the RSV recombinant plasmid standards was used to determine the analytical sensitivity of the singleplexrtRAA assay. The reproducibility of the assay was evaluated by testing each dilution eight times. All of the reactions with $1 \times 10^{4}-1 \times 10^{1}$ copies of template were successfully detected. A dilution with one copy of the template was detected six times. The data are shown in Table 2. There was no signal in any of the NTC reactions. A typical sensitivity amplification curve of this assay for RSV is shown in Fig. 1.

Seventy-seven clinical specimens from patients with other respiratory virus infections were used to evaluate

Table 2 The reproducibility of singleplex-rtRAA and duplex-rtRAA

Copies/reaction

No. of positive sample/no. of samples tested by the rtRAA assay for detection of $\mathrm{RSV}^{\mathrm{a}}$

\begin{tabular}{ll}
\hline RSV singleplex-rtRAA & $\begin{array}{l}\text { RSV } \\
\text { duplex- } \\
\text { rtRAA }\end{array}$
\end{tabular}

\begin{tabular}{lll}
\hline 10000 & 8 & 8 \\
1000 & 8 & 8 \\
100 & 8 & 8 \\
10 & 8 & 8 \\
1 & 6 & 5 \\
\hline
\end{tabular}

${ }^{a}$ Each dilution was tested in a total of 8 replicates the specificity of this assay. The results showed no crossreactions with other respiratory viruses, indicating that the singleplex-rtRAA assay was specific for RSVs (data not shown).

The optimum concentration of the RSVIC was 100 copies for each duplex-rtRAA assay. In eight replicates, RSV plasmids ranging from $1 \times 10^{5}-1 \times 10^{1}$ copies per reaction showed positive results, and five of eight reactions with 1 copy of template were positive for RSV (Table 2). These results showed that the duplex-rtRAA assay was able to detect 1 copy per reaction in the presence of 100 copies of RSVIC per reaction. The detection limit of the RSV duplexrtRAA assay at $95 \%$ probability was 5.0 copies per reaction (probit analysis, $p \leq 0.05$ ). The amplification curves of the duplex-rtRAA are shown in Fig. 2A and B. NTC reactions were performed in parallel, and no signal was observed.

A clinical evaluation of both the singleplex-rtRAA assay and duplex-rtRAA assay was performed using 278 clinical specimens of nasopharyngeal secretions. An RT-qPCR assay was performed in parallel. As shown in Table 3, 102 (102/278) RSV specimens were detected by both rtRAA assays, and the results were in agreement with those obtained by RT-qPCR, which also showed 102 RSV-positive specimens. The CT values of these 102 samples ranged from 17.90 to 35.63 . The results showed that the sensitivity and specificity of both rtRAA assays was $100 \%$ consistent with RT-qPCR when testing clinical samples. IC amplification signals were detected not only with the $102 \mathrm{RSV}$-positive specimens but also with the other 176 non-RSV specimens and with each NTC, indicating that the RSVIC worked effectively. The kappa values ( $\kappa)$ for RT-qPCR and singleplexrtRAA or duplex-rtRAA were both $1(p \leq 0.001)$, revealing that there were no significant differences between the two
Fig. 1 The amplification curves of the singleplex-rtRAA. A serial tenfold dilution of RSV recombinant plasmids from $1 \times 10^{4}$ to $1 \times 10^{0}$ copies per reaction was used to determine the detection limit. NTC, notemplate control

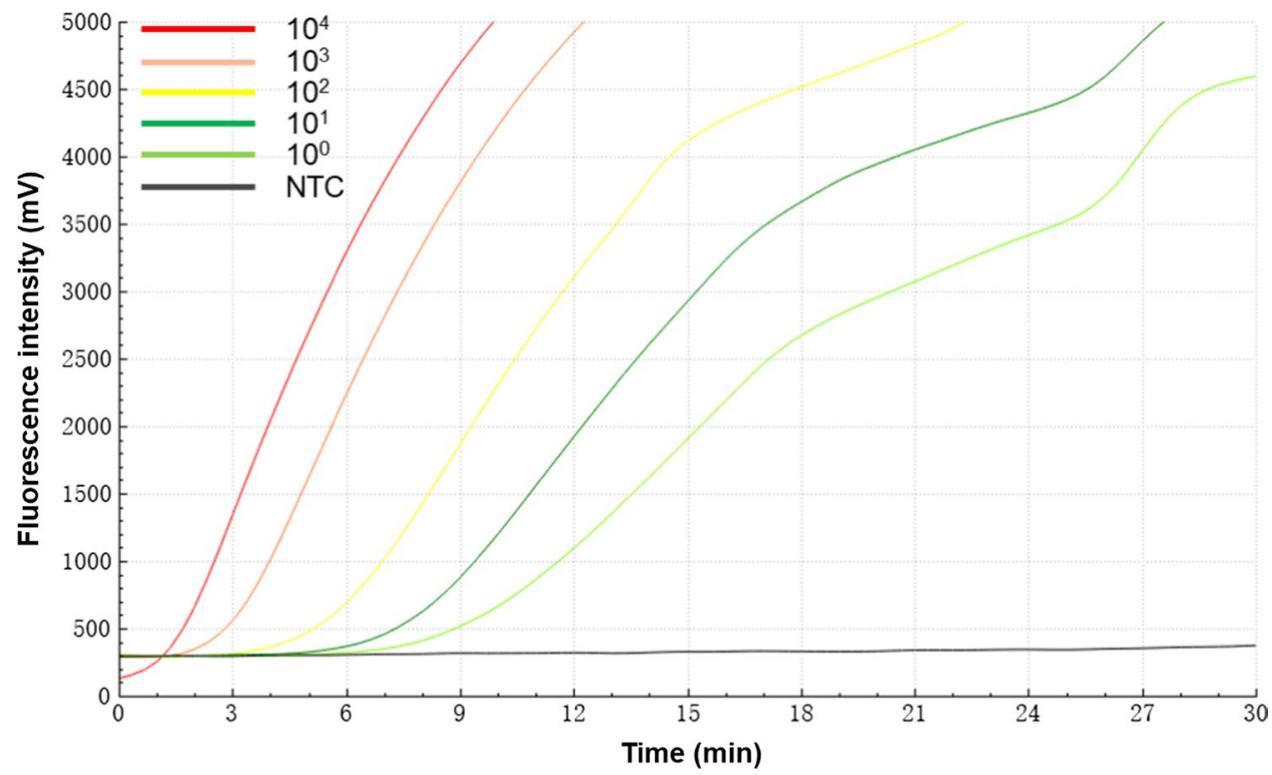


Fig. 2 The amplification curves of the duplex-rtRAA. Successful simultaneous detection of RSV target and the IC template (100 copies per reaction) was observed. (A) FAM fluorescence signal produced by RSV recombinant plasmids (Target) amplification. A higher plasmid concentration resulted in an earlier appearance of an amplification signal in the presence of the IC template (100 copies). (B) HEX fluorescence signal produced by amplification of the internal control template. In the presence of a series of concentrations of RSV plasmids, IC (100 copies) amplification signals appeared at approximately the same time. However, when the RSV plasmid concentration increased, the fluorescence intensity of the IC decreased. In the presence of $10^{4}-10^{0}$ copies of RSV plasmid per reaction, no significant competitive inhibition was observed in the amplification of the IC. In the presence of $10^{5}$ copies of RSV plasmid per reaction, the amplification of IC was significantly inhibited

Table 3 Detection of RSV in clinical specimens

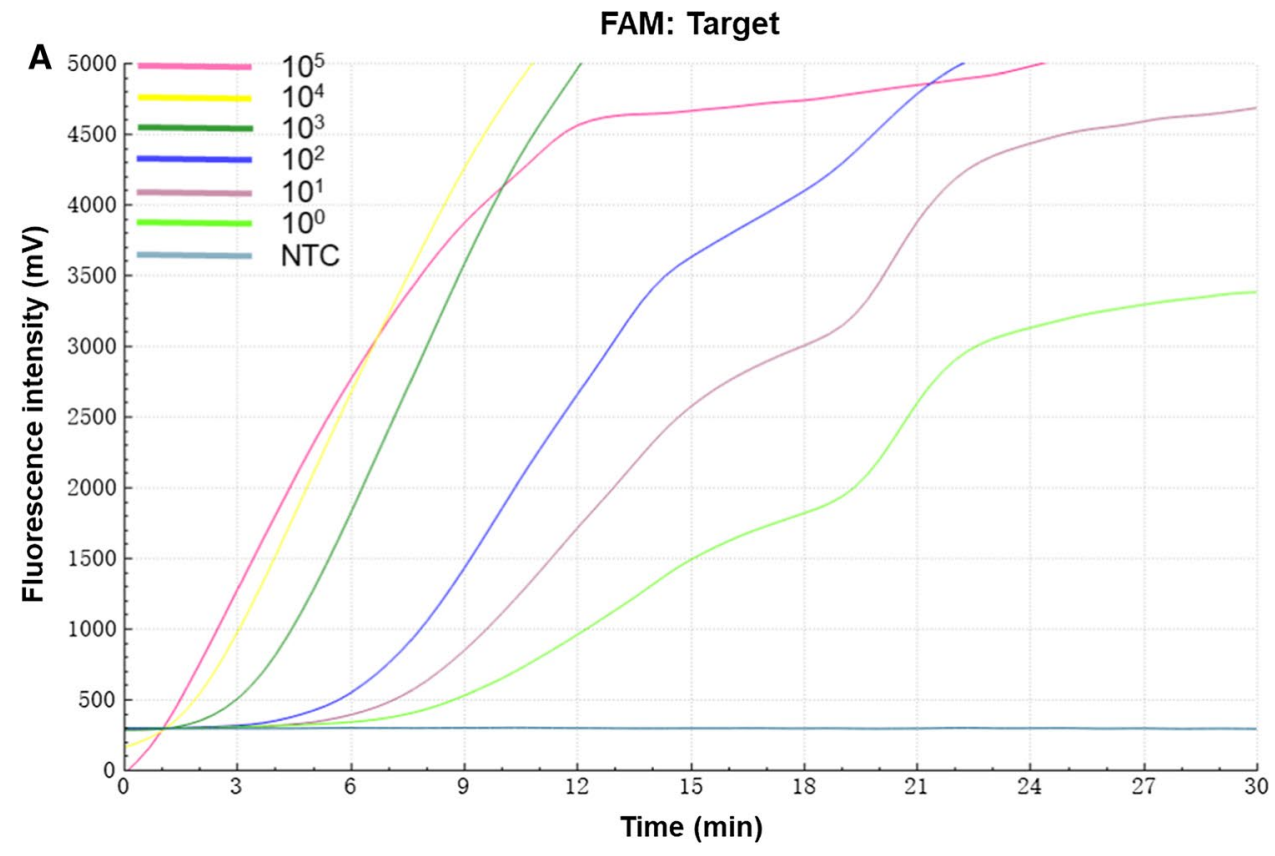

HEX: IC

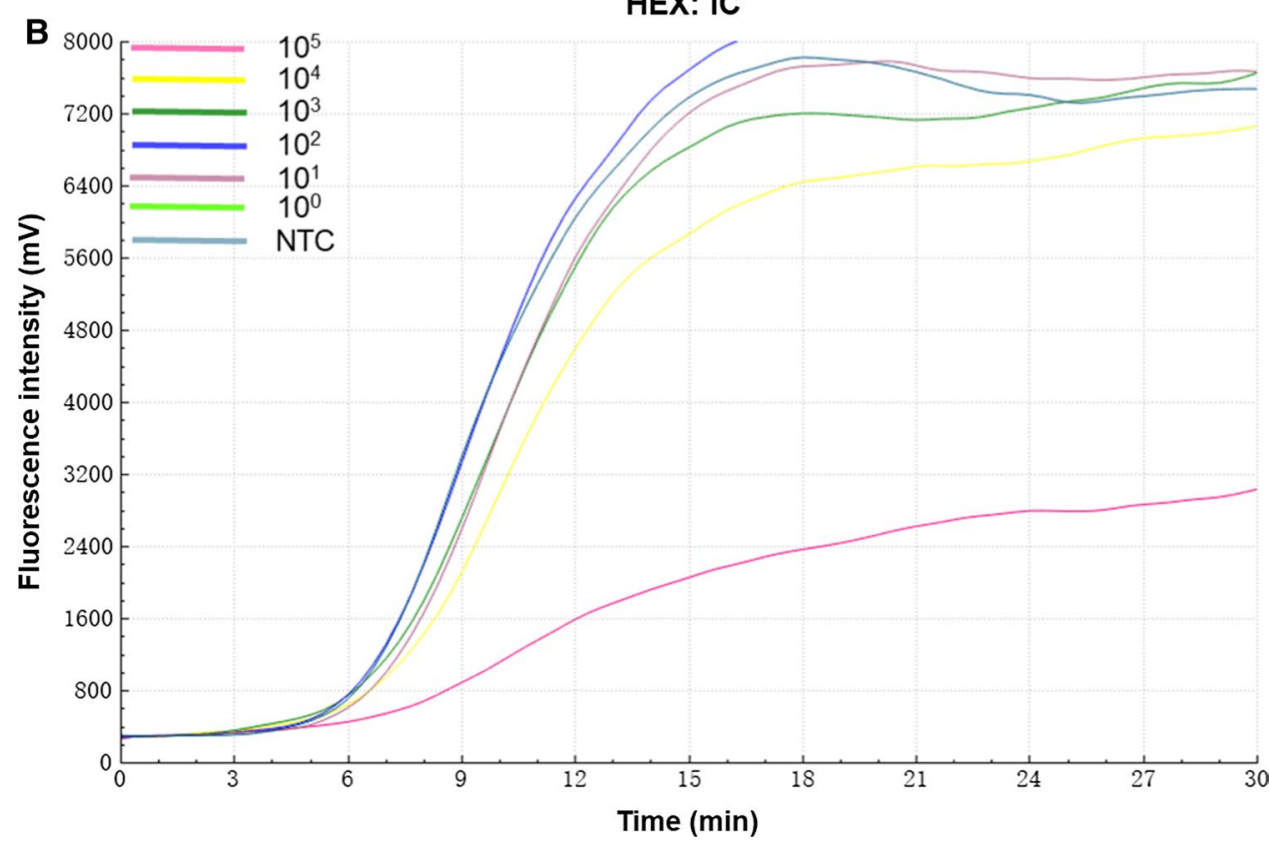

\begin{tabular}{llrrrrr}
\hline Assay & & RT-qPCR & & Kappa & $P$-value \\
\cline { 3 - 5 } & & Positive & Negative & Total & & \\
\hline Singleplex-rtRAA & Positive & 102 & 0 & 102 & 1 & $<0.001$ \\
& Negative & 0 & 176 & 176 & & \\
& Total & 102 & 176 & 278 & & $<0.001$ \\
Duplex-rtRAA & Positive & 102 & 0 & 102 & 1 & \\
& Negative & 0 & 176 & 176 & & \\
& Total & 102 & 176 & 278 & & \\
& & &
\end{tabular}


rtRAA assays and the RT-qPCR assay for detecting RSV. The cycle threshold (CT) values of the RT-qPCR and the threshold time (TT) values of the duplex-rtRAA were well correlated, with an $\mathrm{R}^{2}$ value of 83.1 (Fig. S2).

Rapid and accurate diagnosis of RSV infection is necessary to monitor and control RSV epidemics in clinical settings and to investigate outbreaks. Point-of-care testing (POCT) is no doubt the most powerful tool in this regard because of its advantages of portability of experimental instruments, simple operation and real-time reporting of results. Our duplex-rtRAA assay takes only 20 minutes to obtain a positive result, whereas RT-qRCR usually requires 1-3 hours. Moreover, PCR also requires a professional operator, a special experiment configuration, and thermocycling equipment to achieve high precision, which are major obstacles to its application in the field for POCT. In the present study, the duplex-rtRAA assay for RSV achieved a level of sensitivity and specificity comparable to that of RT-qPCR. The characteristics of rapidity, portability of the instrument, labor saving, and low cost, combined with the use of lyophilized reagents, which are convenient for storage and transport, give the duplex-rtRAA assay great potential for application in the field for POCT for clinical diagnosis of RSV.

To some extent, the presence of nucleic acid inhibitors limits, the applicability of nucleic acid tests (NATs, such as PCR and isothermal amplification technology) by significantly reducing their sensitivity and amplification efficiency [26] and can even lead to false negative results, which in turn can lead to an erroneous diagnosis. Thus, a reliable detection method is critical for clinical diagnosis. In our previous studies, the development of RAA assays for the detection of several pathogens, such as the RSV-A and -B [27], coxsackievirus A10 and coxsackievirus A6 [28], and single-nucleotide polymorphisms (SNPs) [29] only focused on detection of pathogens but did not successfully apply an internal control to monitor the reaction system. Since the RAA reaction is an enzymatic reaction controlled by a variety of enzymes, it is more susceptible to the influence of inhibitors in the specimen. Different from previous studies, we added an IC to achieve optimal conditions for experimental operation and to monitor possible inhibitory reactions in the experiment, in order eliminate false-negative results and to improve the reliability of the assay. There are two main types of ICs: competitive and non-competitive. Non-competitive ICs require different primers and probes for each reaction, and due to the differences in the primer sequences, amplification of non-competitive sequences may not accurately reflect the amplification of the primary target [30]. In order to avoid the risk of unnecessary interactions of multiple primers, we used a competitive IC method to amplify the target and IC with a set of common primers in the same reaction tube under the same conditions. In this case, the only sequence difference between the RSV sequence and RSVIC sequence was in the probe-binding region. In contrast to another reported competitive IC method [31], this strategy allowed the RAA to amplify the target and RSVIC simultaneously, ensuring to the maximum extent the same competitiveness and amplification efficiency. Normally, a competitive IC reduces the efficiency of amplification of the target, which in turn affects the sensitivity of detection. In the duplex-rtRAA assay, we determined the optimal concentration of IC (100 copies per reaction). At this concentration, the stability of the duplexrtRAA assay was confirmed, while the sensitivity of detection of the RSV was not affected. The interpretation of the results obtained using the duplex-rtRAA assay is as follows: 1) If both the target pathogen and IC are amplified, the result should be regarded as positive. 2) If the IC is amplified but the target pathogen is not, this result should be regarded as negative. 3) If the target pathogen is amplified but the IC is not, the result should be regarded as positive because this result might be due a the high concentration of target pathogen competitively inhibiting the amplification of the IC [30]. 4) If neither the target pathogen nor the IC is amplified, the result indicates that the reaction was inhibited and the test results for this sample are invalid [30].

Previous studies have shown that the $\mathrm{L}$ polymerase gene is highly conserved among RSV strains [32,33]. Our rtRAA assay for detecting RSV was also developed targeting this locus and both the singleplex-rtRAA assay and the duplexrtRAA assay showed excellent sensitivity, reproducibility and specificity. The sensitivity of the duplex-rtRAA assay was 5.0 copies per reaction as determined by probit analysis of the results of eight runs, which is sufficiently sensitive for detection. Our specificity test was performed by testing 176 clinical specimens containing non-RSV respiratory pathogens. No nonspecific amplification or detection was found, indicating that there was no cross-detection of other respiratory viruses. In order to verify the diagnostic applicability and the clinical effectiveness of the methods established in this study, 278 clinical specimens of nasopharyngeal aspirates were tested simultaneously by the two rtRAA assays and RT-qPCR, which was used as a reference. The results from the two assays were $100 \%$ consistent with those from RT-qPCR, indicating that the performance of both methods is satisfactory. Therefore, we believe that our duplex-rtRAA is a reliable method for identification of RSV.

Our research needs further improvement. First, all of the specimens in this study were nasopharyngeal aspirates collected from children. Different types of specimens still need to be tested to verify the applicability of our duplexrtRAA assay. Second, the multiplex amplification of RAA with internal control needs further evaluation using a larger sample size.

In summary, a duplex-rtRAA assay incorporating an internal control was successfully developed and evaluated using clinical samples for rapid identification of RSV with 
high sensitivity and specificity. The inclusion of an internal control provides a valuable reference for practical applications. The duplex-rtRAA assay is expected to be an attractive alternative to traditional laboratory-based molecular diagnostic methods in low-resource settings.

Acknowledgements We acknowledge the Children's Hospital of Hebei Province, China, for providing clinical specimens.

Funding This work was supported by grants from China Mega-Project for Infectious Disease (2018ZX10711001, 2017ZX10104001 and 2017ZX10302301-004-002). A Chinese patent is pending.

\section{Compliance with ethical standards}

Conflict of interest All the authors approved the final manuscript and they have no conflict of interest to declare.

Ethical approval All aspects of the study were performed in accordance with national ethics regulations and approved by the Institutional Review Boards of National Institute for Viral Disease Control and Prevention, Center for Disease Control and Prevention of China.

\section{References}

1. Ye S, Wang T (2018) Laboratory epidemiology of respiratory viruses in a large children's hospital. Medicine 97:e11385

2. Englund JA, Sullivan CJ, Jordan MC, Dehner LP, Vercellotti GM, Balfour HH (1988) Respiratory syncytial virus infection in immunocompromised adults. Ann Intern Med 109:203

3. Falsey AR, Treanor JJ, Betts RF, Walsh EE (1992) Viral respiratory infections in the institutionalized elderly: clinical and epidemiologic findings. J Am Geriatr Soc 40:115-119

4. Chen J, Hu P, Zhou T, Zheng T, Zhou L, Jiang C, Pei X (2018) Epidemiology and clinical characteristics of acute respiratory tract infections among hospitalized infants and young children in Chengdu, West China, 2009-2014. BMC Pediatr 18:216

5. Mufson MA, Örvell C, Rafnar B, Norrby E (1985) Two distinct subtypes of human respiratory syncytial (RS) virus. Virus Res $3: 2111$

6. Mazur NI, Higgins D, Nunes MC, Melero JA, Langedijk AC, Horsley N, Buchholz UJ, Openshaw PJ, Mclellan JS, Englund JA (2018) The respiratory syncytial virus vaccine landscape: lessons from the graveyard and promising candidates. Lancet Infect Dis. https://doi.org/10.1016/S1473-3099(18)30292-5

7. Barenfanger J, Drake C, Leon N, Mueller T, Troutt T (2000) Clini$\mathrm{cal}$ and financial benefits of rapid detection of respiratory viruses: an outcomes study. J Clin Microbiol 38:2824

8. Rogers BB, Shankar P, Jerris RC, Kotzbauer D, Anderson EJ, Watson JR, O'Brien LA, Uwindatwa F, Mcnamara K, Bost JE (2015) Impact of a rapid respiratory panel test on patient outcomes. Arch Pathol Lab Med 139:636

9. Jonathan N (2006) Diagnostic utility of BINAX NOW RSV—an evaluation of the diagnostic performance of BINAX NOW RSV in comparison with cell culture and direct immunofluorescence. Ann Clin Microbiol Antimicrob 5:13

10. Reis AD, Fink MC, Machado CM, Paz Jde PJP Jr, Oliveira RR, Tateno AF, Machado AF, Cardoso MR, Pannuti CS (2008) Comparison of direct immunofluorescence, conventional cell culture and polymerase chain reaction techniques for detecting respiratory syncytial virus in nasopharyngeal aspirates from infants. Revista Do Instituto De Medicina Tropical De São Paulo 50:37

11. Borek AP, Clemens SH, Gaskins VK, Aird DZ, Valsamakis A (2006) Respiratory syncytial virus detection by Remel Xpect, Binax now RSV, direct immunofluorescent staining, and tissue culture. J Clin Microbiol 44:1105-1107

12. Falsey AR, Formica MA, Walsh EE (2002) Diagnosis of respiratory syncytial virus infection: comparison of reverse transcriptionPCR to viral culture and serology in adults with respiratory illness. J Clin Microbiol 40:817-820

13. Bonroy C, Vankeerberghen A, Boel A, Beenhouwer HD (2010) Use of a multiplex real-time PCR to study the incidence of human metapneumovirus and human respiratory syncytial virus infections during two winter seasons in a Belgian paediatric hospital. Clin Microbiol Infect 13:504-509

14. Mentel R, Wegner U, Bruns R, Gürtler L (2003) Real-time PCR to improve the diagnosis of respiratory syncytial virus infection. J Med Microbiol 52:893-896

15. Yan L, Zhou J, Zheng Y, Gamson AS, Roembke BT, Nakayama S, Sintim HO (2014) Isothermal amplified detection of DNA and RNA. Mol Biosyst 10:970-1003

16. Compton J (1991) Nucleic acid sequence-based amplification. Nature 350:91-92

17. Deiman B, Schrover C, Moore C, Westmoreland D, Wiel PVD (2007) Rapid and highly sensitive qualitative real-time assay for detection of respiratory syncytial virus A and B using NASBA and molecular beacon technology. J Virol Methods 146:29-35

18. Notomi T, Okayama H, Masubuchi H, Yonekawa T, Watanabe K, Amino N, Hase T (2000) Loop-mediated isothermal amplification of DNA. Nucleic Acids Res 28:E63

19. Hoos J, Peters RM, Tabatabai J, Grulichhenn J, Schnitzler P, Pfeil J (2017) Reverse-transcription loop-mediated isothermal amplification for rapid detection of respiratory syncytial virus directly from nasopharyngeal swabs. J Virol Methods 242:53-57

20. Vincent M, Yan X, Kong H (2004) Helicase-dependent isothermal DNA amplification. EMBO Rep 5:795

21. Piepenburg O, Williams C, Stemple D (2006) Na: DNA detection using recombination proteins-art. no. e204. PLoS Biol 4:1115-1121

22. Bei L, Cheng HR, Yan QF, Huang ZJ, Shen GF, Zhang ZF, Yinv LI, Deng ZX, Lin M (2010) Recombinase-aid amplification: a novel technology of in vitro rapid nucleic acid amplification. Sci Sin 40:983-988

23. Zhang X, Guo L, Ma R, Cong L, Wu Z, Wei Y, Xue S, Zheng W, Tang S (2017) Rapid detection of Salmonella with recombinase aided amplification. J Microbiol Methods 139:202-204

24. Wang J, Xu Z, Niu P, Zhang C, Zhang J, Guan L, Kan B, Duan Z, Ma X (2014) A two-tube multiplex reverse transcription PCR assay for simultaneous detection of viral and bacterial pathogens of infectious diarrhea. Biomed Res Int 2014:648520

25. Sanghavi SK, Arlene B, Shahid H, Rinaldo CR (2011) Clinical evaluation of multiplex real-time PCR panels for rapid detection of respiratory viral infections. J Med Virol 84:162-169

26. Rådström P, Löfström C, Lövenklev M, Knutsson R, Wolffs P (2003) Strategies for overcoming PCR inhibition. CSH Protoc 2008:pdb.top20

27. Chen C, Li XN, Li GX, Zhao L, Duan SX, Yan TF, Feng ZS, Ma $\mathrm{XJ}$ (2018) Use of a rapid reverse-transcription recombinase aided amplification assay for respiratory syncytial virus detection. Diagn Microbiol Infect Dis 90:90-95

28. Yan TF, Li XN, Wang L, Chen C, Duan SX, Qi JJ, Li LX, Ma XJ (2018) Development of a reverse transcription recombinase-aided amplification assay for the detection of coxsackievirus A10 and coxsackievirus A6 RNA. Arch Virol 163:1455-1461

29. Duan S, Li G, Li X, Chen C, Yan T, Qiu F, Zhao L, Zhao M, Wang L, Feng Z (2018) A probe directed recombinase amplification 
assay for detection of MTHFR A1298C polymorphism associated with congenital heart disease. Biotechniques 64:211

30. Hoorfar J, Malorny B, Abdulmawjood A, Cook N, Wagner M, Fach P (2004) Practical considerations in design of internal amplification controls for diagnostic PCR assays. J Clin Microbiol 42:1863-1868

31. Higgins O, Clancy E, Forrest MS, Piepenburg O, Cormican M, Boo TW, O'Sullivan N, Mcguinness C, Cafferty D, Cunney R (2018) Duplex recombinase polymerase amplification assays incorporating competitive internal controls for bacterial meningitis detection. Anal Biochem 546:10-16

32. Mu Y, Zeng J, Chen Q, Jia L, Wang L, Yao F, Meng C, He Z, Zhang C, Ming X (2014) New method for the visual detection of human respiratory syncytial virus using reverse transcription loop-mediated amplification. J Virol Methods 206:84-88

33. Eugene-Ruellan G, Freymuth F, Bahloul C, Badrane H, Vabret A, Tordo N (1998) Detection of respiratory syncytial virus A and B and parainfluenzavirus 3 sequences in respiratory tracts of infants by a single PCR with primers targeted to the L-polymerase gene and differential hybridization. J Clin Microbiol 36:796

Publisher's Note Springer Nature remains neutral with regard to jurisdictional claims in published maps and institutional affiliations. 